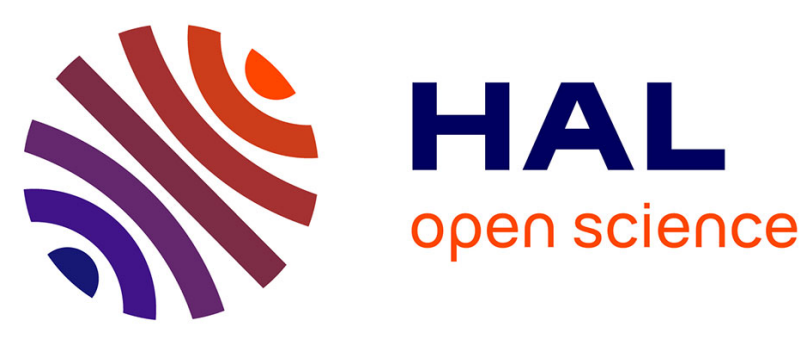

\title{
L'émancipation aujourd'hui
}

\author{
Fabrice Flipo
}

\section{To cite this version:}

Fabrice Flipo. L'émancipation aujourd'hui. Revue du MAUSS, 2011, 38, pp.369-384. 10.3917/rdm.038.0369 . hal-00958068

\section{HAL Id: hal-00958068 https://hal.science/hal-00958068}

Submitted on 11 Mar 2014

HAL is a multi-disciplinary open access archive for the deposit and dissemination of scientific research documents, whether they are published or not. The documents may come from teaching and research institutions in France or abroad, or from public or private research centers.
L'archive ouverte pluridisciplinaire HAL, est destinée au dépôt et à la diffusion de documents scientifiques de niveau recherche, publiés ou non, émanant des établissements d'enseignement et de recherche français ou étrangers, des laboratoires publics ou privés. 


\title{
L'émancipation aujourd'hui
}

\author{
Fabrice Flipo
}

\section{L'ÉMANCIPATION MODERNE}

Jusqu'à la moitié du $\mathrm{xx}^{\mathrm{e}}$ siècle, domine une conception claire de l'émancipation. Elle consiste pour l'essentiel en l'universalisation du modèle moderne de société. Une société moderne, c'est une société fondée sur l'échange, la division du travail, la différenciation sociale, la « solidarité organique », l'individu et l'individualisme. Par contraste, une société « primitive», c'est une société dans laquelle domine la communauté, l'autoproduction, la polyvalence des personnes, dont aucune n'est détentrice d'un savoir-faire véritablement spécifique, la «solidarité mécanique » et «l'individu collectif». Le "développement» désigne le passage d'un état à l'autre, le second étant seul considéré comme véritablement humain, celui qui permet de développer pleinement les facultés humaines.

Ces idées s'élaborent au seuil de la modernité. Pour Kant, à la fin du $\mathrm{xVIII}^{\mathrm{e}}$ siècle, l'histoire est ouverte. La «perfectibilité », objet des Lumières, est un possible dont le contenu ne peut être déterminé que par l'action collective, la raison n'ayant qu'un usage "régulateur». Les Physiocrates ont certes mis en avant quelques principes «d'économie », analysant les flux de biens et de monnaie de manière à démontrer comment les faire augmenter. Mandeville et bien d'autres, dont Voltaire, ont cherché à démontrer la supériorité des valeurs bourgeoises, des arts mécaniques, du luxe, de l'industrie sur la guerre, la dépense ostentatoire, les arts libéraux et l'ascétisme religieux. Mais ce sont des options politiques parmi d'autres.

Hegel les élève au rang de vérité de l'humanité. Et il inaugure le recours systématique au concept de « développement» (Entwicklung). L'organisation sociale moderne devient l'aboutissement de l'odyssée de l'Esprit. Être humain, véritablement humain, c'est être situé à la pointe la plus extrême du « développement » de l'Esprit qui, chez Hegel, prend la forme de la société moderne. Après des siècles de progrès, c'est là qu'elle est la plus auprès d'ellemême. Hegel concède encore un soutien à la monarchie mais, comme le remarque le jeune Marx, ce n'est pas là que mène sa philosophie. Désigner le chef de l'État par un principe naturel, l'hérédité, est totalement contraire au reste de l'œuvre de Hegel, dont l'effort est au contraire de ramener la nature, principe étranger, toujours menaçant, à l'humanité [Marx, 2010, p. 84]. Hegel aurait dû aboutir à identifier humanisme et naturalisme, l'unité aveugle originelle trouvant, au terme de l'histoire, sa plénitude.

Hegel fait l'objet de deux lectures qui symbolisent les deux modalités sous lesquelles l'humanité va "se développer », toutes deux fondées sur l'échange économique. La première se fonde sur le libre marché. C'est « la fortune », dit Hegel, qui préside à la répartition de la propriété [ibid., p. 84]. Le libéralisme reprend cette idée et la généralise. Hegel est le premier à intégrer les idées d'Adam Smith et celle des « économistes » (les Physiocrates) dans une théorie complète de l'État. Il met en avant le rôle essentiel des fonctionnaires, encore souligné de nos jours par Marcel Gauchet. Le libéralisme permet la croissance optimale de l'échange et de la division du travail, qui engendrent à leur tour richesse et progrès technique : "l'homme » acquiert des pouvoirs nouveaux, il va sur la Lune, se déplace à des vitesses jamais vues. Les propos de Hegel ne sont pas isolés, ainsi chez J. B. Say :

« La perfection de nos outils est liée à la perfection de notre espèce. C'est elle qui établit la différence qu'on observe entre nous et les sauvages. » [Say, 1996, p. 287].

Alfred Marshall, en 1906, se référant largement à Hegel [Marshall, 1906, Introduction], oppose les sociétés traditionnelles, qui sont des « sociétés de caste », dans lesquelles les professions sont fixes et souvent héréditaires, aux sociétés modernes, qui sont plastiques et évolutives [ibid., tome I, p. 8]. L'évolution part donc d'une situation dans laquelle l'homme est confronté à une nature hostile et aboutit à une autre situation dans laquelle la nature est dominée. Les « sociétés primitives » sont donc, comme le soutient Durkheim, l'humanité réduite à son expression la plus épurée, la plus simple [Durkheim, 2008, p. 2]. Elles sont le prototype de «l'ancêtre commun» à toutes les sociétés « développées ». De ce point de vue, une société primitive est équivalente à une autre, les différences telles que l'art, la religion ou la culture peuvent être considérées comme secondaires. Toutes tendent à se développer, comme le suggère Adam Smith :

« Suivant le cours naturel des choses, la majeure partie du capital d'une société naissante se dirige d'abord vers l'agriculture, ensuite vers les manufactures, et en dernier lieu vers le commerce étranger. Cet ordre de choses est si naturel, que dans toute société qui a quelque territoire, il a toujours, à ce que je crois, été observé à un certain point. » [Smith, 1995, livre III, chapitre 1]. 
développement contient des particularités. Woodrow Wilson, au lendemain de la guerre, appelait déjà à respecter le « développement politique » propres aux différentes régions du monde, notamment la Russie bolchevique -, mais il appelait aussi à respecter la liberté de commerce, condition indispensable au développement.

L'autorégulation d'une société civile mue par le profit est moins au programme d'Adam Smith que de celui de Malthus. C'est donc avec raison que les socialistes vont le prendre pour cible pour construire la seconde voie moderne d'émancipation: le socialisme. Ce dernier reprend, le plus souvent de manière indépendante, la critique que Marx adresse à Hegel selon laquelle une économie fondée sur des «lois naturelles» ne peut mener qu'à l'aliénation. Ce « naturel » est le masque de la domination. Une société émancipée doit être libérée de cette aliénation. Pierre Leroux, Étienne Cabet, Charles Fourier, Louis Blanc, Proudhon etc. cherchent à réagir devant la tentative de transformer le travail, l'argent et la terre en marchandises [Polanyi, 1983]. La solution est d'organiser le travail de manière consciente, et non de le laisser aux frasques errements ou extravagances ?? du marché. Les socialistes, jusqu'à aujourd'hui, sont réformistes, «trade-unioniste », espérant le Grand Soir mais ne boudant pas ces victoires immédiates que sont les grandes conquêtes sociales : la limitation de la journée de travail, l'interdiction du travail des enfants etc., bref tout ce qui constitue peu à peu la social-démocratie et qui restaure un contrôle de la société sur sa propre activité, mettant fin à «l'anarchie industrielle », en particulier dans le domaine du travail.

C'est en relisant Hegel, notamment la Logique, à la faveur d'une période de reprise économique et de baisse de l'activité révolutionnaire, que Marx va proposer une troisième voie d'émancipation. Pour Marx, les socialistes sont « utopiques », ils prônent des moyens qui n'ont aucune chance d'aboutir car ils sont fondés sur la volonté, sur des idéaux. Ils ignorent l'histoire. Or « tous les conflits de l'histoire ont leur origine dans la contradiction entre les forces productives [qui tendent à la croissance] et le mode d'échanges » [Marx, 1970, p. 134]. Le passage d'un stade à un autre est dialectique : quelque chose est conservé. Les différents stades se succèdent et sont successivement dépassés, vers une synthèse de plus en plus générale, des formes toujours plus accusées et plus universelles, incarnant mieux «l'homme générique ». Ainsi se forment deux classes, deux communautés, dont l'une seulement est révolutionnaire, porteuse de l'universalité : le prolétariat. D'où cette conclusion. Les communistes :

« [...] ont découvert comment à travers toute l'histoire les individus, en tant qu'“hommes privés", ont été à l'origine de l'“intérêt général". » "Pour nous, le communisme n'est pas un état de choses qu'il convient d'établir, un idéal auquel la réalité devra se conformer. Nous appelons communisme le mouvement réel qui abolit l'état actuel des choses. Les conditions de ce mouvement résultant des données préalables telles qu'elles existent présentement [...] Le prolétariat ne peut exister qu'en tant que force historique et mondiale, de même que le communisme, action du prolétariat, n'est concevable qu'en tant que réalité "historique et mondiale"; existence historique et mondiale des individus, cela veut dire que ces individus mènent une existence qui se rattache directement à l'histoire universelle [...]. » [ibid., p. 174].

Marx et Engels estiment avoir montré que les individus de l'époque actuelle seront contraints d'abolir la propriété privée [ibid., p. 192]. La libération est un fait historique, provoqué par des conditions historiques, et non un fait intellectuel, c'est un fait nécessaire. Dans cette perspective, le théoricien, l'intellectuel n'a qu'une seule place : celui de stratège. Réforme ou révolution? Comment les alliances doivent-elles être passées ? etc. Lénine, Trotski, Kautsky, Rosa Luxemburg, Bernstein et bien d'autres s'affrontent sur ces questions de direction du mouvement.

Ainsi aboutissons-nous à trois modèles principaux, qui ont tous connu une mise en œuvre concrète : libéralisme, socialisme et communisme révolutionnaire.

\section{LA CRISE CONTEMPORAINE}

Ces trois formes entrent peu à peu en crise, au $\mathrm{xx}^{\mathrm{e}}$ siècle, sous l'effet de différents chocs successifs. Le premier choc de grande ampleur est produit par la Seconde Guerre mondiale et le nazisme. L'horreur des camps de concentration, l'énormité d'une guerre industrialisée, déclenchée, de plus, par l'un des pays les plus civilisés de la planète, sème le doute. Les Trente Glorieuses ne parviennent pas totalement à faire oublier ce traumatisme que l'École de Francfort commence à théoriser. Un second choc se produit avec l'effondrement de l'Union Soviétique, en 1989, qui s'accompagne de la libéralisation de pays comme l'Inde ou la Chine, le communisme révolutionnaire se confinant désormais à quelques bastions dont le destin est peu envié, tels Cuba ou la Corée du Nord. Un troisième choc, amorcé dans les années 1960 mais qui n'atteint sa maturité que dans les années 2000, est la crise écologique. Un quatrième choc est encore à venir : c'est celui qui est provoqué par la décolonisation et l'avènement d'une période "postcoloniale » dont la portée ultime est une « provincialisation » de l'Europe [Chakrabarty, 2009] et de la modernité européenne. Ces quatre chocs sont liés entre eux, ils sont les divers aspects d'une seule et même crise : celle de l'universalisme occidental.

La première remise en cause porte sur les Lumières et la raison instrumentale. Adorno propose une première critique de l'industrie culturelle et de la sérialisation qu'elle produit. Avec Horkheimer, il entend questionner la réalité de ces «Autres » de la modernité que sont l'irrationalité, la magie et autres attributs prêtés aux peuples primitifs [Horkheimer \& Adorno, 1974]. La critique principale porte sur l'emballement dont la raison émancipatrice des Lumières aurait été 
victime, appliquant aveuglément la raison instrumentale à tout ce qu'elle touche, jusqu'à produire Auschwitz. Cette critique de la raison instrumentale prend les trois courants d'émancipation classiques dans ses filets, car tous trois s'en réclament, tous trois entendent s'appuyer sur la science, en particulier sur diverses conceptions de « l'économie », pour fonder et orienter leur conception du « faire-société », leur conception de la civilisation. C'est bien au cœur des sociétés libérales et social-démocrates qu'Auschwitz s'est produit. Avec Marcuse, la théorie psychanalytique élaborée par Freud prend une ampleur nouvelle. Le freudo-marxisme trouve, dans les contestations de Mai 68, un terrain privilégié pour s'étendre. Gilles Deleuze et Félix Guattari lui donnent, en France, une profondeur toute particulière. La critique de la rationalité instrumentale donne aussi naissance à la microanalyse du pouvoir élaborée par Michel Foucault, ainsi qu'au M.A.U.S.S., qui s'en prend à « l'utilitarisme » dans sa tentative de réduire les relations sociales au calcul. Ces différents courants ne s'étonnent pas de l'effondrement de l'Union Soviétique, ni des décolonisations. Aucun d'entre eux, toutefois, à part de vagues critiques de « l'instrumentalisation de la nature » (Marcuse), ne voit réellement venir la crise écologique. Si le critère opère, c'est sous une forme très allégée, égocentrique : le « nouvel esprit du capitalisme », dont Luc Boltanski et Ève Chiapello [1999] ont perçu l'essor dans la littérature managériale. Le socialisme se convertit à «l'économie de marché », le Parti communiste français n'en finit plus de mourir et la Ligue communiste révolutionnaire renonce à la dictature du prolétariat.

La critique écologiste se construit largement hors les sciences sociales : ce sont les sciences de la nature qui se rebellent contre le « développement». Pour reprendre l'expression de Bruno Latour, ici ce sont « les faits » qui sont en crise, et non les opinions : le climat change-t-il ? les vaches deviennent-elles folles ? les OGM sont-ils maîtrisés ? etc. Des scientifiques s'élèvent contre d'autres scientifiques, qu'ils accusent de minimiser certaines réalités du monde contemporain. Les best-sellers du mouvement écologiste naissant sont écrits par des naturalistes : Road to Survival (William Vogt, 1948), Our Plundered Planet (Fairfield Osborn, 1948), La Bombe P (Ann et Paul Ehrlich, 1968), Nous n'avons qu'une seule Terre (de l'écologue René Dubos et de l'économiste britannique Barbara Ward, 1972) L'Encerclement (Barry Commoner, 1972), Le Macroscope (Joël de Rosnay, 1975), L'Homme re-naturé (Jean-Marie Pelt, 1977) etc. Ces voix montent en puissance dans les années 1960 et 1970, avant de s'atténuer, de revenir autour de 1988-1990, avec à l'apogée le Sommet de la Terre à Rio, puis de reculer de nouveau, avant de réapparaître au milieu des années 2000. Elles ne veulent pas seulement «protéger la nature » mais fonder un nouveau paradigme dont la nature serait le pivot. Ceci heurte fortement les mouvements d'émancipation plus anciens, car la nature, c'est Malthus, c'est de lieu de l'aliénation, de la primitivité, c'est précisément ce contre quoi ils luttent. Mais ils n'ont pas perçu que le sens du concept a changé et que la nature, pour les écologues et écologistes, ce n'est ni la justification idéologique de la domination bourgeoise, à la manière de Malthus, ni ce lieu hostile et menaçant que nous devons nous empresser d'exploiter pour l'humaniser. C'est un lieu fragile et irremplaçable, dont l'existence est une composante essentielle de notre commune humanité, comme le suggère Hans Jonas [1979].

Ce faisant, la critique écologiste indique des lieux et des processus qui, dans la nature, ont été largement négligés par l'expansion industrielle qui a accompagné toutes les théories modernes du développement : les services rendus par les écosystèmes, le caractère entropique de certains stocks souterrains etc. Pour l'écologie, la nature n'est plus le lieu de la solitude, du travail, mais le lieu de la société. « Le social », pour elle, est fondé sur la coopération universelle de tous les êtres, appartenant ou non à l'espèce humaine, les non-humains jouant un rôle majeur dans la production des biens communs dont nous jouissons (stabilité climatique, fertilité etc.) et dont l'usage massif de ressources fossiles avait pu faire oublier la nécessité. Le «travail », au sens de division du travail et d'échange, n'est donc plus le lieu unique de la société. Les humains ne sont plus les seuls avec qui faire société est possible. À l'inverse, la société industrielle, qui envisage la vie et la diversité biologique comme un frein à l'expansion de sa conception de la société, apparait comme l'adversaire à contrer. Cette forme de société ne paraît plus être un progrès mais un monstre mécanique, anonyme, immaîtrisable, broyant le vivant et appauvrissant le monde, provoquant la mort partout où elle passe. Les sociétés peu développées apparaissent comme ayant su conserver sagesse et division raisonnable du travail. La critique écologiste paraît donc "réactionnaire », au sens strict du terme : elle appelle à un retour en arrière, du point de vue des développementistes.

Mais du point de vue de l'écologie politique, c'est aller ailleurs qui est en jeu. La remise en cause est profonde, car tout, dans la société industrielle, dépend de la division du travail : les hautes technologies etc. mais aussi les sciences humaines, divisées en disciplines et en sous-disciplines, les ministères etc. La critique écologique désacralise ce que l'homme moderne considère comme son être intime, son accomplissement, sa vérité : c'est en cela que Hegel est pertinent, dans cette analyse. L'écologie est un mouvement épistémologique : «Comment ne le serait-elle pas ?» [Moscovici, 1978, p. 100]. Les écologistes paraissent aussi réactionnaires aux libéraux qu'aux socialistes et aux communistes. Dans le même temps, avec leur souci de mettre en avant un monde interconnecté, dans lequel la propriété privée semble devoir être abolie, avec la force avec laquelle ils entendent transformer le monde, sous le vert paraît se cacher le rouge. Brun ou rouge ? La sociologie des écologistes en perd son vocabulaire. Et la sociologie écologiste affirme ne plus n'être qu'une antisociologie, cette dernière n'étant, à ses yeux, que la justification du développement : « La sociologie s'est constituée comme l'idéologie de la modernité [...] la sociologie s'est définie spontanément comme l'étude de la société, acceptant même que ce mot s'entende comme la société moderne, par opposition à « communauté », type d'organisation définissant les sociétés anciennes. » [Touraine, 1984, p. 21]. L'écologie politique 
n'oublie pas les inégalités, elle les pluralise, les multiplie. La lutte des classes n'est plus centrale. Pour l'écologie politique, elle ne l'a jamais été.

C'est la pensée de Jacques Derrida qui est mobilisée, à l'échelle internationale, pour entrer dans l'époque « postcoloniale », qui est encore largement ignorée des anciennes métropoles, trop confiantes dans leur propre grandeur. Émergent dans la littérature des métropoles, le mouvement est déjà très affirmé dans les anciennes colonies, au grand désespoir des trois mouvements d'émancipation cités qui sont implantés dans ces pays. L'Inde est, dans ce contexte, un exemple intéressant à plus d'un titre. C'est un pays qui est souvent considéré comme étant le plus éloigné de l'Occident, en termes culturels. Louis Dumont [1979; 2008], comme beaucoup d'autres, voyait dans le système des castes l'exact opposé de la quête individualiste moderne : un ensemble de droits et de devoirs ordonnés par une relation hiérarchique stable, car ancrés dans un ordre sacré, indiscutable et immuable, déterminant jusqu'aux professions des personnes. L'historiographie et la sociologie indiennes ont relativisé ces catégories, montrant notamment comment la dimension religieuse des castes avait été renforcée par les Anglais eux-mêmes, car elle leur permettait d'en marginaliser les revendications politiques [Khare, 2006; Gupta, 2006]. Amartya Sen [2006] a revendiqué l'existence d'une Inde fondée sur l'argumentation, dont il situe l'apogée à l'époque mongole. Des historiennes ont cherché à établir que les fameuses « lois de Manou », qui ont servi à des générations d'anthropologues pour interpréter la société indienne, n'ont jamais été réellement appliquées [Thapar, 2003]. Des intellectuels indiens postmarxistes ont fondé les Subaltern Studies, un collectif dont l'ambition était de parvenir à écrire une histoire de l'Inde "par en bas », loin des récits issus de la puissance colonisatrice, mais aussi loin du récit des élites nationalistes, jugées trop occidentalisées [Nandy, 2006]. Le collectif, fondé dans les années 1980, s'est délité, mais la problématique postcoloniale n'a cessé de gagner en profondeur, en quête d'un universel qui n'aurait pas été imposé par en haut.

Ces trois pensées critiques de la modernité peuvent être dites postmodernes ou antimodernes, ce qui explique deux choses : qu'elles soient perçues par les trois courants émancipateurs comme incarnant la Réaction, ou risquant de l'incarner; et qu'elles offrent toutes une certaine porosité face à la Réaction, aux mouvements qui considèrent que la solution réside dans la soumission à un ordre éternel. Pourquoi ? Parce qu'elles soutiennent toutes les trois que la modernité n'est pas la vérité de l'homme, et mettent par là en évidence la dimension métaphysique de la modernité. Or les Modernes se croient, précisément, débarrassés de toute métaphysique, de toute religion, de toute extériorité. La modernité, c'est la forme de société dans laquelle l'homme coïncide avec lui-même, comme le disait Hegel, et les trois courants émancipateurs parlent eux aussi de la même chose. Or les trois courants vont contre ces thèses, ils « déconstruisent » les concepts de la modernité pour démontrer leur caractère aliénant : aliénation de la nature, dont nous sommes abusivement coupés ; aliénation des colonisés, pris dans une histoire qui se fait sans eux ; aliénation des sujets, à qui une raison mutilante est imposée, au mépris de leurs désirs authentiques, au mépris de leur nature véritable. Les débats autour de la décroissance, en France, sont tout à fait symptomatiques de cette situation, mais nullement singuliers. Ce n'est donc pas seulement l'écologie qui manque d'une théorie alternative du lien social, comme cela lui est fréquemment reproché, mais la modernité dans son ensemble. À ce titre, les critiques de la modernité devraient prendre conscience qu'elles sont logées à la même enseigne, bien qu'elles explorent des voies différentes.

Or nous sommes face à des défis terribles. L'année prochaine, en 2012, à Rio, se négociera le « développement durable », autrement dit la mesure dans laquelle la vérité de l'homme doit être amendée pour prendre en compte les critiques qui lui ont été faites. Certaines critiques sont dévastatrices, elles remettent en cause la validité même du « développement», montrant par exemple qu'il n'est pas envisageable de généraliser le «mode de vie » des pays développés. Les sous-développés doivent-ils rester dans leur inhumanité ? Ou doit-on revoir le modèle ? Attribuer la faute aux riches ou au capitalisme ne suffit pas. Que les riches deviennent pauvres ne conduit pas à changer le modèle. Que le profit soit aboli, que la propriété privée soit « socialisée » ne change pas davantage la configuration générale du développement, qui continue de se concevoir comme échange croissant, division du travail, et n'accepte de prendre en compte les contextes locaux que sous la forme de «traditions» ou de «folklore » subsistant à côté de la modernité triomphante. En aucun cas, ces « traditions » ne peuvent représenter un modèle alternatif de société.

Les négociations sont mal parties : au lieu de parler du «développement», on ne va parler que «d'économie verte ", c'est-à-dire de partage de ce qui reste à exploiter sur la planète. Et, dans ce contexte, la question posée par Malthus est de nouveau à l'ordre du jour, et certains n'y vont pas par quatre chemins : "L'élimination nucléaire instantanée des centres de population pourrait même être considérée comme une délivrance, comparée aux famines et aux massacres se prolongeant pendant des décennies. À la longue, probablement avant 2150, la population mondiale sera tombée à un niveau que les énergies renouvelables, principalement la biomasse, pourront soutenir. Il est probable qu'elle soit similaire à la population d'avant la Révolution industrielle. » [Stanton, 2005].

\section{Quel uniVersalisme?}

Nous ne pouvons pas renoncer à une certaine universalité, ne serait-ce que parce que l'humanité ne s'est jamais trouvée avoir à faire des choix dont la portée aura été aussi universelle. Sans références universelles, comment trouver 
une solution aux négociations commerciales, à la question du changement climatique etc. ? Comment distinguer une solution émancipatrice d'une solution qui ne serait que le fruit de rapports de force ? D'une dynamique de l'identité, du particularisme?

Une première piste consiste à renvoyer ces débats au verdict de la démocratie, en s'en tenant à en discuter les conditions. C'est ce qu'a proposé la seconde génération de l'École de Francfort, en particulier Habermas. Il n'est plus question de définir un universel, ce qui est à l'agenda est de discuter de son contenu. C'est aussi ce que propose Rawls, d'une certaine manière, avec sa «théorie étroite (thin) du bien ». C'est un «minimum d'unanimité », que toutes les philosophies morales et politiques supposent [Rawls, 1997, p. 305]. Cette théorie étroite comprend les libertés de base et les biens qui sont nécessaires pour les mettre en œuvre. Elle n'inclut pas l'ensemble des projets rationnels de vie, qui restent au libre choix des agents. Elle défend une sorte de socle minimal qui doit être encouragé par les structures de base de la société [ibid., § 65]. On pourrait la compléter par la théorie proposée par Bruno Latour [1999], dans laquelle la composition du monde commun est « écologisée ». À ces théories, on peut aisément opposer l'argument marxien du réel contre le formel.

Une seconde approche consiste à s'intéresser de nouveau à l'égalité, dans une approche qui excède la seule dimension économique. À partir du moment où l'on sort de la stricte égalité économique, l'égalité se dit de multiples manières, comme l'a remarqué Amartya Sen [1992]. Ces dernières années, la question de l'égalité s'est centrée sur un nouveau paradigme, dit de la « reconnaissance », inventé par Axel Honneth, et qu'Alain Caillé propose de relier au paradigme du don. Honneth se tourne vers Hegel pour remettre en chantier la question de la reconnaissance sous trois modes : amoureuse, juridique (économique) et culturelle. Cette évolution correspond à la montée des «politiques de l'identité » (genre, origine, religion etc.) qui semblent s'imposer dans les années 1980 et 1990, au détriment des revendications économiques sur lesquelles les trois courants émancipateurs modernes étaient bâtis. Comme le suggère Nancy Fraser [2004], les deux perspectives, économique et de reconnaissance, sont rarement séparées dans les cas concrets d'oppression. Et elles ne l'ont jamais été : les revendications ouvrières, au XIX ${ }^{e}$ siècle, portaient au moins autant sur l'estime et la reconnaissance des compétences que sur la dimension économique. L'originalité de ces courants est plutôt d'insister sur des dimensions de l'égalité qui avaient été négligées. De nombreux écrits socialistes se soucient du statut des femmes. Plus originale est la question des minorités sexuelles, mais aussi moins prégnante, numériquement. Fraser propose le concept de "parité de participation », qui implique égalité de ressources et égalité de respect [ibid., p. 161]. Cela permet en effet de restituer une dimension émancipatrice, qui rejoint la conception du développement d'Amartya Sen [2000], comme liberté.

Ces questions, toutefois, restent largement à côté de la question écologique, qui pose la question d'un rapport émancipateur à la nature, c'est-à-dire au monde commun. Elles peuvent donc rester compatibles avec le paradigme classique de la croissance : une croissance féministe, égalitaire, tiers-mondiste, bref « qualitative »; mais une croissance quand même. D’ailleurs, la croissance a toujours été « qualitative », la productivité du travail demande des sciences de la nature, des juges, des instituteurs etc.

Il est urgent de «changer le gâteau ». L'écologie politique peut y conduire. Collaborer avec le vivant est généralisable à la planète entière, dans le principe. Reste à savoir ce que cela recouvre exactement. Les écologistes ont largement exploré cette alternative, mais ils n'ont pas de réponse toute faite. Le recours à des techniques « écologiquement intensives » permet de se passer des fossiles tout en nourrissant la population. Les low tech permettent de satisfaire des besoins tels que le déplacement ou l'habitat avec une moindre consommation d'énergie, une moindre «empreinte écologique », ce qui laisse autant de place disponible pour les autres, humains et non-humains. Égalité : que chacun ait droit à un même « espace écologique » [Flipo, 2007] ! Comme le suggère Bernard Perret [2011], l'écologie sera la nouvelle rationalité, elle remplacera la rationalité économique. Un point important, toutefois, est que cette science ne doit pas être appropriée par un corps de savants qui seraient détachés de la société. Les critères d'une action «écologique » doivent donc être établis par en bas et non par en haut, ce qui serait gros d'une nouvelle technocratie. Les écologistes y ont pensé, Serge Moscovici [2002, p. 197] allait jusqu'à dire que l'écologie scientifique doit être la fille de l'écologie militante et non l'inverse. Cet objectif peut être atteint en mettant les experts au service des citoyens, plutôt que l'inverse ; ainsi, le savoir se coconstruira collectivement. Le but de l'écologie n'est pas un État global. Ce n'est pas « une société planétaire mais une planète de sociétés » [Moscovici, 1978, p. 53]. Même Edward Goldsmith [1992, p. 81] reconnaît que « l'écologie est subjective ».

Et les rapports de force ? Cet aspect est très bien explicité dans un petit modèle proposé par Ramachandra Guha et Madhav Gadgil [2005], qui distingue trois classes : les « omnivores », qui vivent plutôt dans les villes et mangent des ressources qui proviennent du monde entier ; les «peuples des écosystèmes », qui vivent dans les lieux où sont prélevés les ressources pour les omnivores et où ceux-ci déposent leurs déchets (y compris leurs gaz à effet de serre) ; et les réfugiés écologiques, qui se trouvent chassés des lieux vivables par les excès des omnivores. L'intérêt des « peuples des écosystèmes » s'est manifesté, à Cochabamba et ailleurs, en réclamant que la nature soit dotée de droits, et en souhaitant être responsables de leur mise en œuvre. Ces droits n'impliquent pas de position "préservationniste », contrairement à ce à quoi renvoie le plus souvent la thématique des « droits de la nature » en Occident [Stone, 1972]. Ce n'est pas la nature vierge qui est à préserver : c'est la fertilité et l'équilibre de notre corps inorganique, dont nous 
sommes évidemment partie. C'est une attitude qui rejoint la Déclaration des droits de la nature, discutée en ce moment en Bolivie et à l'ONU. La fertilité résulte de la capacité d'une culture à percevoir les fins réseaux de vie qui tissent le milieu, pour les faire fructifier et s'épanouir. Ainsi pouvons-nous jouir de leurs fruits. A contrario, l'utilisation massive de ressources minérales pour pallier la destruction de la vie est mortifère.

\section{REFAIRE DE L'HISTOIRE, DÉFENDRE L'ÉGALITÉ}

Ce programme n'implique pas de « retour en arrière », contrairement aux courants qui tendent à idéaliser le passé ou les sociétés « primitives ». Mais pour pouvoir faire face à ces dérives, les raisons d'une telle idéalisation, fréquemment observée, doivent être comprises.

La raison la plus évidente est que le passé, les sociétés sous-développées et les sociétés primitives sont les grandes figures de la non-modernité. C'est contre elles que la modernité s'est construite. À ce titre, elles ne sont que le produit de la modernité, pas son envers, ni son dehors, ou du moins pas davantage qu'une société aussi peu primitive que la société chinoise ou indienne. Les sociétés primitives ne sont donc pas plus notre passé que notre avenir, elles ne sont $a$ priori ni émancipatrices ni oppressantes, ne pouvant pas être aussi facilement jugées et mises dans un même sac. Le propos vaut aussi pour le passé, ou plus exactement pour l'immense ensemble des sociétés qui ne sont ni « développées » ni «primitives », et que l'on tend facilement, en Occident, à ramener à notre Moyen Âge, qui était particulièrement oppressant, à certaines époques.

Dans une phase historique qui voit la modernité entrer en crise, les personnes cherchent des ressources dans ce qui est son Autre : la non-modernité. Et, jusqu'à un certain point, la démarche est valide, car elle permet de dépoussiérer le regard que nous, Modernes, portons sur le monde. Ainsi, découvre-t-on que les Orientaux peuvent faire la révolution, que les peuples «primitifs » détiennent bien un savoir-faire écologique [Harding, 1996] etc. Le communisme primitif devient désirable parce qu'il est l'extrême inverse de la modernité avancée, d'où les écovillages, idéal qu'on retrouve mis en avant jusque dans le très médiatisé rapport de Tim Jackson [2010] sur une prospérité sans croissance.

La voie de salut est bien de ce côté-là, mais en maintenant une dimension critique, qui évite de se contenter d'inverser les choses, de jeter le bébé avec l'eau du bain. Dans tous les cas, la solution est de refaire de l'histoire, de la géographie, avec une distance critique. Voyager, refuser le localisme identitaire, intolérant, ou pratiquant une tolérance isolationniste, un faux pluralisme, critiqué par Isabelle Stengers [2003]. Mais des superstitions bien connues de notre Moyen Âge, au premier chef l'Inquisition et ces procès d'insectes dont Luc Ferry se moquait, on ne peut en déduire le caractère intrinsèquement oppresseur de ce que l'ONU qualifie de sociétés « en développement ».

Peut-on encore avoir une lecture dialectique de l'histoire ? Le point n'est pas à écarter. Le rôle de médiateur dialectique ne peut plus être l'expansion des forces productives industrielles. Mais que penser de l'appropriation des niches écologiques, qui est finalement au fondement des comportements prédateurs ? Elle pourrait provoquer une tendance à la paupérisation qui deviendra d'autant plus marquée que les ressources souterraines vont s'épuiser. Et serait grosse de révolutions à venir.

\section{Références bibliographiques}

Boltanski L., Chiapello E., 1999, Le Nouvel esprit du capitalisme, Gallimard, Paris.

Chakrabarty D., 2009, Provincialiser l'Europe. La pensée postcoloniale et la différence historique, Amsterdam, Paris.

Dumont L., 2008 (1977), Homo Aequalis, t. I, Gallimard, Paris.

- 1979 (1967), Homo hierarchicus, Gallimard, Paris ;

Durkheim E., 2008 (1912), Les Formes élémentaires de la vie religieuse, PUF, Paris.

Flipo F., 2007, Justice, nature \& liberté, Parangon, Lyon.

Fraser N., 2004, «Justice sociale, redistribution et reconnaissance », Revue du Mauss semestrielle, $\mathrm{n}^{\circ} 23$, p. 152164.

Goldsmith E., 1994 (1992), Le défi du 21e siècle, Éditions du Rocher, Paris.

Guha R. \& Gadgil M., 2005, Ecology \& equity, in Guha R. \& Gadgil M., The Use and Abuse of Nature, Oxford University Press, USA.

Gupta D., 2006, Anti-Utopia. Essential writings of Andre Beteille, Oxford University Press, USA.

Harding S., 1996, "La science moderne est-elle une ethnoscience ?», in WAAST R. (dir.), Les Sciences hors l'Occident au $x x^{e}$ siècle, ORSTOM, Paris, p. 239-261.

Horkheimer M. \& Adorno T., 1974, La Dialectique de la raison, Gallimard, Paris.

Jonas H., 2008 (1979), Le Principe responsabilité, Flammarion, Paris.

Khare R.S., 2006, Caste, Hierarchy, And Individualism: Indian Critiques Of Louis Dumont's Contributions, Oxford University Press, Delhi.

Latour B., 1999, Politiques de la nature, La Découverte, Paris. 
Marshall A., 1906, Principes d'économie politique, Consulté sur Gallica <http://gallica.bnf.fr/>.

Marx K., 2010, Critique du droit politique hégélien, Éditions Allia, Paris.

- 1970, Idéologie allemande, Éditions Sociales, Paris.

Moscovici S., 2002, De la Nature, Métaillé, Paris.

Moscovici S., in Ribes J.-P., 1978, Pourquoi les écologistes font-ils de la politique?, Le Seuil, Paris.

Nandy A., 2006, The Intimate Enemy. Loss and Recovery of the Self Under Colonialism, Oxford University Press, USA.

Perret B., 2011, Pour une raison écologique, Flammarion, Paris.

Polanyi K., 1983 (1944), La Grande transformation, Gallimard, Paris.

Rawls J., 1997 (1971), Théorie de la justice, Le Seuil, Paris.

Say J.-B., 1996 (1821), Lettres à Malthus, in Cours d'économie politique, Flammarion, Paris.

Sen A.K., 2006, The Argumentative Indian, Penguin Books India, Delhi.

— 2000 (1999), Un Nouveau modèle économique, Odile Jacob, Paris.

- 1992, Inequality Reexamined, Clarendon Press, Oxford.

Smith A., 1995 (1776), Enquête sur la nature et les causes de la richesse des nations, PUF, Paris.

Stanton W., 2005, item 524, ASPO Newsletter $n^{\circ} 52$.

Stone C.D., 1972, « Should trees have standing ? Toward legal rights for natural objects », Southern Californian Law Review, $\mathrm{n}^{\circ}$ 45, p. 450-501.

Thapar R., 2003, History of early India, Penguin Books India, Delhi.

Touraine A., 1984, Le Retour de l'acteur, Fayard, Paris. 